Canadian

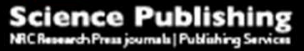

Canadian Journal of Civil Engineering Revue canadienne de génie civil

\title{
Development and Evaluation of a Microscopic Overtaking Gap Acceptance Model for Two-lane Highways
}

\begin{tabular}{|r|l|}
\hline Journal: & Canadian Journal of Civil Engineering \\
\hline Manuscript ID & cjce-2015-0371.R1 \\
\hline Danuscript Type: & Article \\
\hline Complete List of Authors: & $\begin{array}{l}\text { Ghods, Amir H.; University of Waterloo, Civil and Environmental } \\
\text { Engineering } \\
\text { Saccomanno, Frank; University of Waterloo, Civil and Environmental } \\
\text { Engineering }\end{array}$ \\
\hline Keyword: & two-lane highways, overtaking, gap acceptance \\
\hline \multicolumn{2}{|c}{} \\
\hline
\end{tabular}




\title{
Development and Evaluation of a Microscopic Overtaking Gap Acceptance Model for Two-lane Highways
}

\author{
Amir H. Ghods \\ (Corresponding Author) \\ $\mathrm{PhD}$, Research Associate \\ Department of Civil and Environmental Engineering \\ University of Waterloo \\ 200 University Avenue West \\ Waterloo, Ontario, Canada N2L3G1 \\ Tel: +1-519-888-4567, Ex. 33872, Fax: +1-519-888-4349 \\ Email: ahghods@uwaterloo.ca
}

Frank F. Saccomanno

$\mathrm{PhD}$, Professor

Department of Civil and Environmental Engineering

University of Waterloo

200 University Avenue West

Waterloo, Ontario, Canada N2L3G1

Tel: +1-519-888-4567, Ex. 32631, Fax: +1-519-888-4349

Email: saccomano@uwaterloo.ca

Number of Words: 5874

Number of Figures: 6

Number of Tables: 6

Total Word Count: 8874

Accepted for publication in the Canadian Journal of Civil Engineering (CJCE)

March 21, 2016 


\begin{abstract}
A microscopic gap acceptance model is presented that simulates overtaking behavior on two-lane highways. The decision to initiate overtaking is expressed as a function of each driver's perception of the expected Time-to-Collision (TTC) with the nearest opposing vehicle at the end of maneuver. The available gap is accepted if the driver's perception of TTC exceeds a predetermined threshold for safe return (critical TTC). The gap acceptance model is calibrated and validated based on overtaking videorecording data for a two-lane highway. The overtaking gap acceptance model is then compared, for consistency and transferability, with independent aggregate field data, as well as with two other simulation models and values given in the Highway Capacity Manual for similar two-lane highways. The gap acceptance simulation demonstrated that the proposed overtaking model is able to provide reliable measures of traffic attributes for two-lane highway operation, as verified experimentally.
\end{abstract}

Keywords: Two-lane highways, overtaking, gap acceptance 


\section{INTRODUCTION}

In recent years, microscopic simulation models have gained increased acceptance as reliable investigative tools for understanding traffic operations and evaluating safety performance. Despite this trend, the development and application of these models to two-lane highway operations has not kept pace with those of freeways and urban networks, and this is due in large part, to difficulties in modeling the overtaking process. Various methods have been investigated for modeling the overtaking gap acceptance logic in traffic simulation. As compared to car-following or lane-changing models, however, it has been difficult to provide specific calibration parameters for overtaking gap acceptance due to the complexity of the process. This process is rendered complex by the large number of inter-related factors that need to be considered by the overtaking driver in a bi-directional driving regime.

Three major microscopic traffic simulation models have been developed specifically for two-lane highway operations, i.e. TWOPAS (St John and Harwood 1986; Leiman et al. 1998); TRARR (Troutbeck 1981; Shepherd 1994; Hoban et al. 1991), and VTI (Ahman 1972). TWOPAS was developed by MRI (Midwest Research Institute) in the early seventies and was modified by Leiman et al. 1998 under the name of UCBRURAL. This model was applied to estimate two-lane highway capacity and level of service in the Highway Capacity Manual (HCM) (HCM 2000). TWOPAS model is also used in the Interactive Highway Safety Design Model (IHSDM) (Paniati and True 1996) as a Traffic Analysis Module (TAM). The overtaking logic in TWOPAS is probabilistic in nature and is based on gap acceptance probability functions for different overtaken vehicle speeds and sight distance limitations, i.e. limited by opposing vehicle or geometry. The probability functions in TWOPAS were determined from empirical overtaking data collected in the early seventies as reported in Harwood et al. 1999.

TRARR was developed by the Australian Road Research Board (ARRB) as a research tool for the design of passing lanes in level highway segments (Hoban et al. 1991; Lovell et al. 1993). The overtaking logic in TRARR is deterministic and the decision to overtake is based on the available 
overtaking time gap multiplied by a vehicle-specific safety factor. A driver aggressiveness factor is assigned to each vehicle such that drivers do not overtake when their aggression level is lower than that for any driver vehicle(s) in the lead position(s). The application of TRARR in the Netherlands resulted in higher overtaking frequencies or rates when compared to field observations (Hegeman 2004).

The VTI model was developed to analyze traffic behavior for rural two-lane highways in Sweden. The stochastic overtaking logic of VTI is generally more advanced than either TWOPAS or TRARR. It accounts formally for a large number of factors affecting overtaking such as type of overtaking (flying or accelerated), available gap with opposing vehicles, type of overtaken vehicle (car or truck), as well as road cross-sectional width and grade. A total of 32 combinations of these factors were considered by the VTI model for which a separate gap acceptance versus gap size function was developed. Given the large number of factors that could affect overtaking, it is expected that the VTI model requires a significant amount of field data for its calibration and validation. A modified version of VTI known as RuTSim was proposed for two-lane highways by adding intersection control logic to the simulation framework (Tapani 2005); however, the same VTI overtaking model was used. An application of RuTSim for the analysis of overtaking assistance systems is discussed in Hegeman et al. 2009. Among other models, Farah et al. 2009a used a critical gap acceptance concept and a binary Logit choice gap acceptance formulation. The critical gap for an overtaking vehicle was determined based on limited traffic variables, road geometry, and driver characteristics as obtained from a driving simulator. Farah et al. 2009b also developed a model linking overtaking time-to-collision (TTC) to overtaking gap acceptance. Their model is able to control for a myriad of road, traffic, and driver characteristics, as obtained from a driving simulator application.

More recently, Li and Washburn 2011 implemented a two-lane highway overtaking algorithm into CORSIM (Halati et al. 1997). The overtaking decision in this model is based on comparing available gaps with estimates of the safe Passing Sight Distances (PSD) as proposed by the AASHTO Green Book (AASHTO 2004). This approach, although simple to implement, does not make use of any overtaking field data for calibration; nor does it suggest a new behavioral overtaking logic. A number of studies have 
suggested that PSD values in AASHTO are very conservative for application to overtaking behavior (Harwood et al. 2008). Although the model proposed by Li et al. also considered PSD values from the Manual of Uniform Traffic Control Devices (MUTCD), there was no attempt to show which PSD value yielded the more realistic results. Kim and Elefteriadou 2010 made use of the AASHTO Green Book guidelines to develop an overtaking simulation model known as TWOSIM for application to two-lane highway capacity analysis.

This review of current overtaking models indicates that the main challenge in modeling the overtaking maneuver is to provide a sound reliable link between the "decision-to-overtake" and available gaps in the traffic stream subject to changing road and traffic conditions. Unlike other driving regimes (such as car-following or lane-changing), for overtaking gap acceptance, the specification of model parameters are difficult to extract for two-lane traffic conditions and this is due mainly to the need to specify numerous factors influencing gap-acceptance behavior, such as, the size of the available gaps with opposing vehicles, vehicle operating speeds, type and length of vehicles, type of overtaking (flying versus accelerated), and driver's aggression level.

In most of the existing overtaking models the decision to overtake is established as a function of the available gap size (separating overtaking vehicle from the opposing vehicle prior to initiating the overtake). This decision will vary depending on other influencing factors, for instance, the probability of gap-acceptance for a given gap size will depend on the speed of the overtaking vehicle, the speed of the vehicle being overtaken as well as the speed of opposing vehicle. Given the number of these factors and their range of likely values, updating existing simulation models would require extensive overtaking field data, and this becomes especially important since the data used for calibrate most of the existing overtaking models is quite dated now. Hence the approach taken in this paper is to rationalize the data input requirements of the gap acceptance model within a new logic-based framework for two-lane operations. 
The purpose of the research discussed in this paper is to develop and calibrate a new behavioral overtaking gap acceptance model for two-lane highways (referred to as OTSIM). The structure of the proposed model is such that the overtaking influencing factors are mechanistically encapsulated into a new decision variable known as Time-to-Collision (TTC) at the end of the overtaking maneuver as estimated by the driver at the beginning of the maneuver. The new decision variable considers not only the available gap size, but also a wider spectrum of gap acceptance decision variables that results in fewer number of gap acceptance model calibration parameters . TABLE 1 provides a side-by-side comparison of the OTSIM gap acceptance logic and its calibration effort versus those of the existing models.

\section{FORMULATION OF OVERTAKING GAP ACCEPTANCE MODEL}

The gap acceptance logic introduced in this paper takes a mechanistic view of the overtaking process. In this process, the initial desire-to-overtake is triggered in a car-following regime by the following vehicle (FV) driver, when his/her desired speed becomes significantly higher than the operating speed of the lead vehicle (LV) or the vehicle to be overtaken. As reported in Kim and Elefteriadou 2010, the proposed algorithm makes use of a default speed differential of $8 \mathrm{~km} / \mathrm{h}$ to trigger this "desire to overtake". The FV driver searches for an appropriate gap with respect to the opposing vehicle (OP) to safely initiate the maneuver (i.e. pull out into the opposing lane and begin to pass). In principle it is expected that safety in overtaking depends on the FV driver's "perception" of what would be the likely time interval separating its position (post-overtaking) from that of the OP when the overtaking maneuver has been completed. It is reasonable to assume, that the FV driver will need to be cognizant of this "safe separation" in order to avoid a potential head-on crash with the OP. In this paper, this separation gap is referred as the FV's "time-to-collision" or TTC, a measure that encapsulates a full spectrum of physical variables influencing the gap acceptance process. This gap is subject to driver perception at the instant overtaking is initiated by the FV. 
In other words, the perceived $T T C$, in addition to the initial available gap size, is taking into account the effect of overtaking distance and the distance travelled by the opposing vehicle in initiating the overtaking. This technically takes into account a whole spectrum of gap acceptance influencing factors such as the size of the available gaps with opposing vehicles, vehicles operating speeds, type, and length, type of overtaking (flying versus accelerated), as well as single or multiple vehicles overtakes.

In the overtaking gap acceptance logic two measures of TTC need to be considered: 1) that which is perceived by the FV driver prior to initiating the maneuver $\left(T T C^{p}\right)$ and 2) that which is measurable (TTC) from the position and speeds of the relevant vehicles after the overtaking vehicle returns to the original travel lane. Since it is not possible to obtain an accurate estimate of $\operatorname{TTC}^{p}$, it is assumed that $T T C^{p}$ can be expressed as a random variable with a mean of TTC (the actual FV-OP separation time gap at the end of maneuver) and a random error term $(\varepsilon)$, such that:

$$
T T C^{p}=T T C+\varepsilon
$$

Eq. 1

In Eq.1, it is assumed that $\varepsilon$ is a random variable with a normal distribution, such that: $\varepsilon \sim N\left(0, z^{2}\right)$

The assumption of normality and value of the variance term $z^{2}$ must be verified empirically based on observed traffic data.

\subsection{Estimating TTC}

As illustrated in FIGURE 1, the estimation of TTC involves calculation of three sequential overtaking distances (or overtaking phases).

$D_{1}$ : Distance travelled by the FV from initial decision-to-overtake to pull out (beginning of the OT maneuver)

$D_{2}$ : Distance travelled by the FV from pull-out to the point where desired overtaking speed is achieved, and 
$D_{3}$ : Distance travelled by the FV to achieve safe separation with the overtaken vehicle after returning to normal travel lane.

and $D$ : Distance between FV and OP when a gap becomes available

To estimate $D_{1}$, a perception/reaction time of $t_{1}=1 s$ and a constant initial FV speed of $v_{n}^{\text {ini }}$ are assumed such that the overtaking FV vehicle will cover a distance of:

$$
D_{1}=v_{n}^{i n i} \times t_{1}
$$

During this time, the LV is assumed to cover a distance of $D_{1}^{\prime}$ assuming a constant speed of $v_{n-1}$, such that:

$$
D_{1}^{\prime}=v_{n-1} \times t_{1}
$$

To estimate $D_{2}$, the time for the FV to pull out and attain its desired overtaking speed $\left(v_{n}^{\text {des-ov }}\right)$ is estimated as:,

$$
t_{2}=-\frac{v^{\max }}{k a^{\max }} \times \ln \left(\frac{v^{\max }-v_{n}^{\text {des-ov }}}{v^{\max }-v_{n}^{\text {ini }}}\right)
$$

Eq. 4

where

$v_{n}^{\text {des }-o v}=$ desired overtaking speed of the FV

$v^{\max }=$ maximum achievable speed of the $\mathrm{FV}$ (vehicle specific)

$a^{\max }=$ maximum achievable acceleration of the FV from stopped position (vehicle specific)

$k=$ proportion of maximum acceleration employed by the driver for overtaking

The distance covered in interval $t_{2}$ is estimated as:

$D_{2}=v^{\max } t_{2}+\frac{v^{\max }}{k a^{\max }}\left(v^{\max }-v_{n}^{\text {ini }}\right)\left(e^{-\frac{k a^{\max }}{v^{\max }} t_{2}}-1\right)$

Eq. 5

and the corresponding distance traversed by the LV during the $t_{2}$ interval is: 


$$
D_{2}^{\prime}=v_{n-1} \times t_{2}
$$

Eq. 6

The derivation of time duration and distance covered by overtaking vehicle to reach the desired overtaking speed is provided in Appendix 1.

To estimate $D_{3}$, the distance covered by the FV in passing the LV and completing the OT in the interval $t_{3}$, we use the expression:

$$
D_{3}=t_{3} \times v_{n}^{d e s-o v}
$$

Eq. 7

where, $t_{3}$ is obtained from the expression involving headways, length of vehicles, speeds and distances, such that:

$t_{3}=\frac{H W^{r e t}+L_{n-1}+H W^{\text {init }}+L_{n}-\left(D_{1}+D_{2}-D_{1}^{\prime}-D_{2}^{\prime}\right)}{v_{n}^{\text {des-ov }}-v_{n-1}}$

Eq. 8

where:

$H W^{\text {init }}=$ initial distance headway between front bumper of the FV and rear bumper of LV $H W^{\text {ret }}=$ distance headway for pull back (rear bumper of FV and front bumper of LV).

$L_{n-1}=$ length of overtaken vehicle

$L_{n}=$ length of overtaking vehicle

The total overtaking distance and time for FV is estimated as:

$$
\begin{gathered}
D_{O T}=D_{1}+D_{2}+D_{3} \\
T_{O T}=t_{1}+t_{2}+t_{3}
\end{gathered}
$$

The opposing vehicle (OP) is also assumed to maintain a constant speed of $v_{O P}$ during the FV overtaking maneuver. Hence during the $T_{O T}$ interval, the distance covered by the OP is estimated as: 


$$
D_{O P}=T_{O T} \times v_{O P}
$$

Eq. 10

The difference between the initial separation between FV and OP prior to pull out (main gap, $D$ ) and the "closing" distance traversed by these vehicles during the overtaking is referred to as the residual gap $\left(D_{\text {Res }}\right)$, and this gap reflects the distance separating the FV from the OP after overtaking maneuver has been completed (pull back). This is the distance safety margin for avoiding a head-on collision. $D_{\text {Res }}$ can be estimated as:

$$
D_{\text {Res }}=D-\left(D_{O T}+D_{O P}\right)
$$

Eq. 11

TTC is basically $D_{\text {Res }}$ expressed in units of time, such that:

$$
T T C=\frac{D_{R e s}}{v_{O P}+v_{n}^{d e s-o v}}
$$

In those instances where the desired overtaking speed $\left(v_{n}^{\text {des-ov }}\right)$ is not achieved prior to FV pull back, the distance $D_{2}$ is set to the return point and distance $D_{3}$ is set to zero. It should be noted that in the case of multiple overtaking, a contiguous platoon of vehicles (separated by short time headways of less than $3 \mathrm{~s}$ ) can be considered, such that all vehicles in the platoon are assumed to travel at the same speed of $v_{n-1}(t)$ and act as a single "undivided" decision unit or length. Hence the FV driver's initial decision is to overtake all or none of the LV vehicles in the platoon as an undivided unit. In order to generalize the TTC for multiple-vehicle overtakes, the term $L_{n-1}$ in Eq. 8 is replaced with the estimated length of the platoon. This acts to reduce the estimated TTC resulting in a lower overtaking gap-acceptance probability. In the case of a flying overtaking, $v_{n}^{\text {ini }}$ takes the initial non-reduced operating speed of FV, which is normally higher than that of LV. In this case, $D_{2}$ is assumed to be close to zero, since the driver has skipped the catch-up process and the overtaking desired speed is already achieved. This results in reduced overtaking distance and increased TTC. The term TTC in Eq. 12 encapsulates the traffic and physical 
factors, which are assumed to influence overtaking, into a single decision variable. Depending on the parameter values and input variables used in Eqs. 2-12, the resultant TTC can be an accurate time-tocollision or an estimate of perceived time-to-collision. Later on in this paper assumptions are made for some of these parameters and variables, which can be overestimated or underestimated by the driver and results in so-called estimate of driver's perceived TTC.

\subsection{Calibration procedure}

For the overtaking gap acceptance model considered in this paper it is assumed that each driver $(n)$ has a critical minimum acceptable gap (TTC $C_{n}^{\text {crit }}$ ), and this critical gap is assumed to be normally distributed with a mean of $\overline{T T C}^{\text {crit }}$ and a variance of $\sigma^{2}$, such that:

$$
T T C_{n}^{\text {crit }}=\overline{T T C}^{c r i t}+e_{n}
$$

Eq. 13

where, $e_{n} \sim N\left(0, \sigma^{2}\right)$.

The parameters of the distribution for $T T C_{n}^{\text {crit }}$ in Eq. 13 can be determined empirically from observed overtaking data. For accepted gaps, the available $T T C^{p}$ must exceed the critical value for the $n^{\text {th }}$ overtaking driver $\left(T T C_{n}^{\text {crit }}\right)$. Whether the perceived time-to-collision $\left(T T C^{p}\right)$ is accepted or rejected, a decision variable $(y)$ can be defined as series of FV binary decisions, such that for the $n^{\text {th }}$ vehicle in the traffic stream:

$$
y_{n}= \begin{cases}1 & \text { if } T T C^{p} \text { is accepted } \\ 0 & \text { if } T T C^{p} \text { is rejected }\end{cases}
$$

The probability that the $n^{\text {th }}$ overtaking vehicle accepts an available perceived gap $\operatorname{TTC}^{p}$ can be expressed as: 


$$
\begin{aligned}
P_{n}\left(\text { Accept } T T C^{p} \mid \overline{T T C}^{\text {crit }}, \sigma^{2}\right) & =\mathrm{P}\left(T T C^{p}>T T C_{n}^{\text {crit }}\right) \\
& =\mathrm{P}\left(T T C^{p}>\overline{T T C}^{c r i t}+e_{n}\right) \\
& =\varnothing\left(\frac{T T C^{p}-\overline{T T C}^{c r i t}}{\sigma}\right)
\end{aligned}
$$

where, $\emptyset($.$) in the above expression denotes the standard cumulative normal of the Probit function. A$ similar relationship has been applied in the literature to a gap-acceptance problem for stream crossing by Mahmassani and Sheffi 1981 and Daganzo 1981.

The overtaking gap acceptance parameters can be estimated experimentally using maximum likelihood, such that:

$\arg \max L=\prod_{n=1}^{N} P_{n}^{y_{n}}\left(1-P_{n}\right)^{1-y_{n}}$ Eq. 16 $\overline{T T C}^{\text {crit }}, \sigma$

where, $N$ corresponds to the number of vehicles in the traffic stream being considered in "desire-toovertake" mode. Based on the observational overtaking data, Eq. 16 can be solved for $\overline{T T C}^{\text {crit }}$ and $\sigma$, such that the expression $L$ is maximized. For a given distribution of $T T C^{\text {crit }}$, the gap acceptance function is defined as the probability that a randomly selected driver will accept an available "perceived" TTC ${ }^{p}$.

\section{CALIBRATING OVERTAKING GAP ACCEPTANCE}

This section of the paper describes a two-lane highway traffic survey used to collect data for calibrating of the gap-acceptance model, with the subsequent results.

\subsection{Traffic overtaking data}

The overtaking data used to calibrate and validate the gap acceptance model were obtained from a videotaped survey of traffic along a 1-km stretch of two-lane highway in Southern Italy (the SS18 near 
Amantea, CS). The videotaping was carried out over a three-hour period on two consecutive weekdays for ideal uncongested conditions: dry pavement, unrestricted visibility and near zero vertical grade. The posted speed limit on this segment of highway is $80 \mathrm{~km} / \mathrm{h}$. The segment was selected such that overtaking was permitted and geometry did not significantly restrict maximum sight distances. The videotaped segment was situated between two short overpasses.

As illustrated in FIGURE 2, two cameras were located approximately 50 meters above the highway at an offset distance of about 200 meters from the centerline. An average two-way volume of $533 \mathrm{veh} / \mathrm{h}$ was observed for the first day and $436 \mathrm{veh} / \mathrm{h}$ for the second day.

A program was developed by Guido et al. 2013 to extract frame-by-frame video images of the trajectory and speeds of individual vehicles in $0.1 \mathrm{~s}$ intervals as they progress along their travel path. A more detailed description of the data collection experiment has been provided in Guido et al. 2013. For the overtaking model calibration, a total of 97 vehicles trajectories were extracted from the three-hour videotaping in which the potential overtaking vehicle (referred to FV) was assumed to be in "desire-toovertake" mode, although not necessarily in the process of overtaking. To be in desire-to-overtake mode at least one of the following two conditions needs to be met:

- FV accepts an eventual gap within the observed segment after one or more gaps were rejected

- FV is observed to veer toward the centerline, presumably searching for an overtaking opportunity and the headway between the lead and following vehicles is less than 30 meters.

The latter condition was taken from Hegeman et al. 2004, who observed that the distance between the overtaking vehicle and the vehicle being overtaken at the beginning of maneuver is distributed with mean of $17.8 \mathrm{~m}$ and standard deviation of $9.8 \mathrm{~m}$, with about $92 \%$ of headways less than $30 \mathrm{~m}$ in length. The 97 sample trajectories yielded a total of 171 gaps of which 81 were accepted and 90 were rejected.

Using Eq. 2 to Eq. 12, the corresponding perceived time to collision was estimated at the moment the overtaking gaps became available. To estimate $T T C^{p}$ we assumed the following parameters are perceived by the driver to estimate overtaking distance and distance travelled by the opposing vehicle: 
- $\quad v_{n}^{\text {des-ov }}$ is assumed to be $m \mathrm{~km} / \mathrm{h}$ higher than the speed of overtaken vehicle $\left(v_{n-1}\right)$

According to Harwood and Sun 2008, the overtaking speed differential $(m)$ between the FV and LV vehicles can be obtained based on the speed of the LV $\left(v_{n-1}\right)$, such that:

$$
\begin{aligned}
& m=44.1-0.25 v_{n-1} \\
& v_{n}^{\text {des-ov }}=v_{n-1}+m
\end{aligned}
$$

- $\quad$ Return headway $H W^{\text {ret }}$ is assumed to be 1 second as specified by Glennon 1988 .

- Speed of the opposing vehicle $v_{O P}$ is assumed to be the average speed of the traffic stream as observed from the SS18 survey $(90 \mathrm{~km} / \mathrm{h})$.

- $v^{\max }$ is $160 \mathrm{~km} / \mathrm{h}$ for all the vehicles. $k a^{\max }$ was assumed to be $1.82\left(\mathrm{~m} / \mathrm{s}^{2}\right)$. This value is calculated from results of a recent overtaking acceleration study conducted in Brooks 2012. The actual acceleration depends on the speed of overtaking vehicle and can be calculated as: $a(t)=$ $a_{m}\left(1-\frac{v(t)}{v_{m}}\right)$

These assumptions are set to reflect the driver's imperfect judgment of factors affecting the overtaking decision that may result in underestimation or overestimation of actual TTC. The other parameters involved in the calculation of $T T C^{p}$ such as, $D, H W^{\text {init }}, v_{n-1}$, and $v_{n}^{\text {ini }}$ are assumed to be perfectly known by the driver at the beginning of the maneuver. For a given overtaking opportunity in the SS18 videotaped data, each FV driver's $T_{T C}{ }^{p}$ is estimated and gap-acceptance decision with respect to the perceived time-to-collision is recorded as a binary decision variable ( 0 for Rejected, 1 for Accepted). The results of this procedure are presented for a sample of trajectories in TABLE 2.

In this study, it is assumed that overtaking gap decisions are independent events, such that for a single driver the decision to accept a gap is independent of previous rejected gaps. In reality, one can recognize that the decision to accept a gap and overtake is very likely influenced by the number of gaps rejected previously, such that overtaking drivers are subject to an impatience factor in the process that 
increases with the number of gaps that have been rejected. Hence, the higher the number of gaps rejected, the shorter the gap that is eventually accepted to initiate the overtaking. The impatience factor could be obtained by observing sequence of rejected gaps for a longer highway segment observed over a more extensive period of time. However, such data were not available for this study and hence, this term has not been used in this analysis.

The error term associated with $T T C^{p}$, as defined in Eq. 1, can be estimated from observations of accepted overtaking gaps for which TTC can be calculated from the video recorded data at the end of each overtaking maneuver. For the 81 accepted gaps, the difference between the measured valued for the $k^{\text {th }}$ accepted gap at the end of the maneuver $\left(T T C_{k}\right)$ and its perceived value $\left(T T C_{k}^{p}\right)$ at the beginning of the maneuver reflects the error term for the $k^{\text {th }}$ overtaking driver, such that:

$$
\varepsilon_{k}=T T C_{k}-T T C_{k}^{p}
$$

Eq. 18

\subsection{Calibration and validation results}

FIGURE 3 illustrates the distribution of perception errors for a sample of overtaking accepted gaps in the videotaped data. The mean of the perceived distance and time distributions was found to be around zero with corresponding standard deviations of 52 meters and 1.2 seconds, respectively. This also validates the assumption of normality of perception error distribution.

Of the 171 overtaking gap decisions in the videotaped sample, $70 \%$ were selected for calibration and $30 \%$ for validation of the overtaking gap acceptance model. A generalized linear Probit model based on Eq. 15 and Eq. 16 was fitted to the calibration data with the results summarized in TABLE 3. The mean and variance of $T T C^{\text {crit }}$ was estimated from the traffic data to be 3.0 seconds and $0.7 \mathrm{~s}$, respectively, which suggests that $95 \%$ of drivers have critical gap-acceptance thresholds between 1.6 and 4.4 seconds (mean $\pm 2 * \mathrm{SD}$ ). The corresponding distance or headway thresholds range from 80 to 220 meters, between the overtaking vehicle and the opposing vehicle, prior to the overtaking vehicle returning to the normal 
travel lane (assuming average speeds of $90 \mathrm{~km} / \mathrm{h}$ ). These parameters were found to be statistically significant at $95 \%$ confidence level $(\mathrm{p}<0.05)$. The log likelihood value for this expression was found to be -16.35 .

FIGURE 4 illustrates the distribution of $T T C^{\text {crit }}$ superimposed on the Probit cumulative distribution function. The dots along the "1" and "0" lines represent accepted and rejected overtaking gaps, respectively. The negative values for the TTC perceived variable corresponds to unsafe available gaps which would have led to head-on collision if the gap had been accepted.

In order to determine predictive ability of the overtaking gap acceptance model, the Probit model was applied to an "independent" validation sample of gaps (30\% of gaps). The percent correct prediction rate for the validation sample was found to be $89 \%$, confirming that the calibrated model predicts overtaking gap acceptance for a different dataset with very good transferability.

\section{MODEL COMPARISON AND TRANSFERABILITY CHECK}

The above overtaking gap acceptance model was integrated into a more extensive microsimulation framework, that comprises several well-established traffic modules, such as, GHR car following model (Gipps 1981), platoon generation model (Miller 1967), and free-flow driving regime. For the purpose of this study, a computer code was programmed in Matlab to integrate these different modules into a single traffic simulation platform (OTSIM). From the video recording study, the average following time headways for vehicles in desired-to-overtake mode was found to be 0.8 second, while for other car-following drivers not desiring to overtake was $1.3 \mathrm{~s}$. The GHR car-following model parameters were calibrated to address changes in the two car-following regimes. The car-following parameters calibration results are reported in TABLE 4.

FIGURE 5 illustrates the overall structure of the simulation framework. The OTSIM model inputs consist of road, traffic, and vehicle data, as well as behavioral model calibration parameters. The road 
input data includes geometric design features, such as, segment length and grade as well as location of passing/no-passing zones. Traffic input data includes directional flows, proportion of vehicles (passengercar, truck, RV), and distribution of desired speeds. Vehicle data consists of type, weight, length, and engine power as well as vehicle's acceleration and deceleration parameters. OTSIM traffic outputs consist of percent time spent following, average travel speed, journey time, delay, and overtaking frequency. The safety outputs include head-on and rear-end time-to-collision.

In this section of the paper, we test the transferability of the proposed gap acceptance model to independent field data and to simulated results as obtained from TRARR and TWOPAS (IHSDM) models for the same highway segment. The field data and TRARR results were obtained for a five kilometers segment of two-lane highway in the Netherlands, as reported in Hegeman 2004. The posted speed for the Netherland highway is $100 \mathrm{~km} / \mathrm{h}$ for cars and $80 \mathrm{~km} / \mathrm{h}$ for trucks with volumes for all vehicle types of $1026 \mathrm{vph}$ and $471 \mathrm{vph}$ in direction 1 and 2, respectively. A few basic traffic data for the Netherlands highway are given in TABLE 5 .

Since the primary focus of this paper is on overtaking maneuver, the performance measures used in comparing model results are: average overtaking rate (OR) and average travel speed (ATS). For this exercise, it is assumed that the average desired speed of vehicles is equal to the posted differential speed limits of $100 \mathrm{~km} / \mathrm{h}$ and $80 \mathrm{~km} / \mathrm{h}$ for cars and trucks, respectively. The coefficient of variation for the desired speed is assumed to be 0.1 (details about these assumptions are provided in Fitzpatrick et al. 2003).

TABLE 6 provides comparison of OR and ATS between OTSIM and TWOPAS with respect to the field data and TRARR, as reported by Hegeman 2004 for the Netherlands data. TRARR was found to significantly overestimate the overtaking rate in comparison to the field data. However, the OTSIM overtaking rate yielded the lowest error with respect to these data for direction 1. TWOPAS on the other hand generated a significantly higher error (lower overtaking rate) compared to OTSIM for this direction. For direction 2, the lowest error was obtained for TWOPAS; although, the difference between the two 
simulations (TWOPAS and OTSIM) is not high. For ATS, the absolute difference between simulation and the field data was found to be lowest for OTSIM as compared to TWOPAS. It appears that OTSIM was able to yield closer overtaking rates and average travel speed with respect to field data than those that were obtained from the TRARR and TWOPAS model simulations.

A further comparison was made between OTSIM, TWOPAS and values reported in HCM 2010 (HCM 2010) based on the Percent Time Spent Following (PTSF). The PTSF is an indicator of the inability of the FV to overtake so as to attain the desired speed in two-lane traffic operations. As PTSF increases, it is expected that the number of vehicles successfully overtaking to decrease. For this test, simulation results were obtained for a range of analysis direction volumes (100 pc/h to $1700 \mathrm{pc} / \mathrm{h})$ and opposing direction volume (100 pc/h to $1600 \mathrm{pc} / \mathrm{h})$ as illustrated in FIGURE 6.

It can be noted that, at low opposing volumes, where higher passing opportunities are expected, there is a closer match in PTSF between OTSIM and HCM 2010. The TWOPAS results suggest higher values of PTSF and presumably fewer overtaking maneuvers. This is consistent with the findings from of application of OTSIM and TWOPAS to the Netherlands data, as discussed above. As opposing volume increases and passing opportunities are reduced, there is a shift in OTSIM estimates away from the HCM values towards those suggested by TWOPAS. For example, at $1600 \mathrm{pc} / \mathrm{h}$ opposing volume, PTSF obtained from OTSIM matches that from TWOPAS. These results are encouraging in that they suggest an acceptable level of transferability in OTSIM simulation results.

\section{CONCLUSION AND FUTURE WORK}

An analytical and behavioral formulation of the overtaking gap-acceptance process for two-lane highway operations was discussed in this paper. The decision to overtake was expressed as a function of the perceived time-to-collision (TTC) and established driver gap-acceptance thresholds. The gap-acceptance logic adopted in this research is assumed to encapsulate the full spectrum of physical variables influencing the gap-acceptance decision, resulting in reduced numbers of parameters requiring 
calibration. The model was calibrated using observed overtaking data obtained by video-taping a two-lane highway segment. The distribution of critical TTC gaps for a population of drivers was found to be normally distributed with a mean of 3.0 seconds and standard deviation of $0.7 \mathrm{~s}$. The model was then compared with independent aggregate field data as well as simulated results based on TRARR, TWOPAS and HCM models. The overtaking model was found to yield relatively both consistent and transferable results for PTSF, ATS, and overtaking rate when compared to field data and other simulation model values. It must be noted that in this research, the model parameters were determined as per calibration overtaking data for normal driving, road, and traffic conditions as well as good weather and clear visibility. Any changes to these conditions would require re-calibration of overtaking model parameters.

\section{REFERENCES}

AASHTO. 2004. A policy on geometric design of highways and streets. Fifth edition. Washington, D.C.: American Association of State Highway and Transportation Officials.

Ahman, K. I. 1972. Omkorningar av lastbilar. Trafikstudier Statens Vag-Och Trafikinstitut Raport 6.

Brooks, R. M. 2012. Acceleration charachteristics of vehicles in rural pennsylvania. International Journal of Research and Reviews in Applied Sciences 12(3): 449-453.

Daganzo, C. F. 1981. Estimation of gap acceptance parameters within and across the population from direct roadside observation. Transportation Research Part B: Methodological 15 (1): 115.

Farah, H., Bekhor, S., and Polus, A. 2009a. Risk evaluation by modeling of passing behavior on two-lane rural highways. Accident Analysis \& Prevention 41(4): 887-94.

Farah, H., Bekhor, S., Polus, A., and Toledo, T. 2009b. A passing gap acceptance model for two-lane rural highways. Transportmetrica 5(3): 159-72.

Fitzpatrick, K., Carlson, P., Brewer, M. A., Wooldrifge, M. D., and Miaou, S. P. 2003. Design speed, operating speed, and posted speed practices. NCHRP Report 504, Transportation Research Board, Washington, D.C.

Gipps, P. G. 1981. A behavioural car-following model for computer simulation. Transportation Research Part B 15(2): 105-11. 
Glennon, John C. 1988. New and improved model of passing sight distance on two-lane highways. Transportation Research Record: Journal of the Transportation Research Board (1195): 132-7.

Guido, G. P., Vitale, A., Saccomanno, F., Astarita, V., and Giofrè, V. P. 2013. Evaluating accuracy of new algorithm for extracting vehicle tracking data from videotaping. Proceedings of 92nd Annual Meeting of the Transportation Research Board, Washington D.C.

Halati, A., Lieu, H., and Walker, S. 1997. CORSIM-corridor traffic simulation model. Paper presented at the Traffic Congestion and Traffic Safety in the 21st Century Conference, New York.

Harwood, D. W., Gilmore, D. K., Richard, K. R., Dunn, J. M., and Sun, C. 2008. Passing sight distance criteria. NCHRP Report 605, Transportation Research Board, Washington, D.C.

Harwood, D. W., May, A. D., Anderson, I. B., Leiman, L., and Archilla, A. R. 1999. Capacity and quality of service of two-lane highways, NCHRP Final Report. Midwest Research Institute, University of California, Berkeley.

Hegeman, G. 2004. Overtaking frequency and advanced driver assistance systems. Proceedings of IEEE Intelligent Vehicles Symposium.

Hegeman, G., Hoogendoorn, S., and Brookhuis, K. 2004. Observations overtaking manoeuvres on bi-directional roads. Advanced OR and AI Methods in Transportation 505-510.

Hegeman, G., Tapani, A., and Hoogendoorn, S. 2009. Overtaking assistant assessment using traffic simulation. Transportation Research Part C: Emerging Technologies 17(6): 617-630.

Highway Capacity Manual, 2010. Transportation Research Board, National Research Council,Washington D.C.

Highway Capacity Manual, 2000 Transportation Research Board, National Research Council,Washington D.C.

Hoban, C. J., Shepherd, R. J., Fawcett, G. J., and Robinson, G. K. 1991. A model for simulating traffic on two-lane rural roads: User guide and manual for TRARR version 3.2. Paper presented at Australian Road Research Board.

Kim, J., and Elefteriadou, L. 2010. Estimation of capacity of two-lane two-way highways using simulation model. Journal of Transportation Engineering 136(1): 61-66.

Leiman, L., Archilla, R., and May, A. D. 1998. TWOPAS model improvements. University of California, Berkeley. 
Li, J., and Washburn, S. S. 2011. Implementing two-lane highway simulation modeling into CORSIM. Paper presented at 6th International Symposium on Highway Capacity and Quality of Service, Stockholm, Sweden.

Lovell, D. J., LAU, SI, and May, A. D. 1993. Using the TRARR model to investigate alignment alternatives and passing lane configurations on the buckhorn grade. California: Institute of Transportation Studies, UCB-ITS-RR-93-DRAFT.

Mahmassani, H., and Sheffi, Y. 1981. Using gap sequences to estimate gap acceptance functions. Transportation Research Part B: Methodological 15(3): 143-8.

Miller, A. J. 1967. Queueing in rural traffic. in vehicular traffic science. Proceedings of the Third International Symposium on the Theory of Traffic Flow.

Paniati, J. F., and True, J. 1996. Interactive highway safety design model (IHSDM): Designing highways with safety in mind. Paper presented at Roadside Safety Issues Revisited, Irvine, California.

Shepherd, R. 1994. TRARR 4 user manual. Australian Road Research Board Ltd.

St John, AD., and Harwood, DW. 1986. TWOPAS user's guide, a user's guide to TWOPAS-A microscopic computer simulation model of traffic on two-lane, two-way highways. Research, Development, and Technology, FHWA, US Department of Transportation.

Tapani, A. 2005. Microscopic traffic simulation models: Calibration, validation, and computation: Versatile model for simulation of rural road traffic. Transportation Research Record: Journal of the Transportation Research Board (1934): 169-178.

Troutbeck, R. J. 1981. Overtaking behaviour on australian two-lane rural highways. Australian Road Research Board, Special Report SR20. 
TABLE1 Side-by-side comparison of proposed simulation model (OTSIM) versus the existing models

\begin{tabular}{|c|c|c|}
\hline $\begin{array}{c}\text { Model } \\
\text { Name }\end{array}$ & Gap acceptance model description & $\begin{array}{c}\text { Gap Acceptance } \\
\text { Model Calibration } \\
\text { Effort }\end{array}$ \\
\hline TWOPAS & $\begin{array}{c}\text { Stochastic, linking available gaps to probability functions for } \\
\text { different overtaken vehicle speeds and sight distance limitations }\end{array}$ & High \\
\hline TRARR & $\begin{array}{c}\text { Deterministic, based on the available overtaking time gap multiplied } \\
\text { by a vehicle-specific safety factor. }\end{array}$ & Medium \\
\hline VTI & $\begin{array}{c}\text { Stochastic, accounts for a large number of factors affecting } \\
\text { overtaking, such as, type of overtaking (flying or accelerated), } \\
\text { available gap with opposing vehicles, type of overtaken vehicle (car } \\
\text { or truck), as well as road cross-sectional width and grade. }\end{array}$ & Very high \\
\hline $\begin{array}{c}\text { Farah et } \\
\text { al. }\end{array}$ & $\begin{array}{c}\text { Critical gap acceptance concept, based on Time-to-Collision } \\
\text { estimated at the beginning of the overtaking }\end{array}$ & High \\
\hline CORSIM & Based on MUTCD and AASHTO passing sight distance guideline \\
OTSIM & $\begin{array}{c}\text { Critical gap acceptance concept based on estimate of end of } \\
\text { overtaking Time-to-Collision as estimated at the beginning of the } \\
\text { manoeuvre }\end{array}$ & Low \\
\hline
\end{tabular}


TABLE2 A sample of processed disaggregate overtaking data from video cameras

\begin{tabular}{|c|c|c|c|c|c|c|c|c|c|c|c|c|c|c|c|c|c|}
\hline $\begin{array}{c}\text { Vehicle } \\
\#\end{array}$ & $\begin{array}{c}\text { Gap size } \\
\text { (m) }\end{array}$ & $\begin{array}{c}\text { Decision } \\
\text { (0:Reject) } \\
\text { (1:Accept) }\end{array}$ & $\begin{array}{c}\text { Overtaking } \\
\text { veh speed } \\
(\mathbf{k m} / \mathbf{h})\end{array}$ & $\begin{array}{c}\text { Overtaken } \\
\text { veh speed } \\
(\mathrm{km} / \mathrm{h})\end{array}$ & $\begin{array}{c}\text { Opposing } \\
\text { veh speed } \\
(\mathbf{k m} / \mathbf{h})\end{array}$ & $\begin{array}{c}\text { HWinit } \\
(\mathrm{m})\end{array}$ & $\begin{array}{c}\text { HWret } \\
(\mathrm{m})\end{array}$ & $\begin{array}{c}\text { Max } \\
\text { speed } \\
(\mathbf{k m} / \mathbf{h})\end{array}$ & $\begin{array}{c}\mathbf{k}^{*} \mathbf{M a x A c c} \\
(\mathrm{m} / \mathbf{s} 2)\end{array}$ & $\begin{array}{c}\text { OT } \\
\text { desired } \\
\text { speed } \\
(\mathbf{k m} / \mathbf{h})\end{array}$ & $\begin{array}{l}T_{\text {Tor }} \\
\text { (s) }\end{array}$ & $\begin{array}{l}\mathbf{D}_{\text {oT }} \\
(\mathbf{m})\end{array}$ & $\begin{array}{l}D_{\text {op }} \\
\text { (m) }\end{array}$ & $\begin{array}{c}\text { Actual } \\
\text { ResGap } \\
\text { (m) }\end{array}$ & $\begin{array}{c}\text { Perceived } \\
\text { ResGap } \\
\text { (m) }\end{array}$ & \begin{tabular}{|c|} 
Actual \\
TTC (s)
\end{tabular} & $\begin{array}{c}\text { Perceived } \\
\text { TTC (s) }\end{array}$ \\
\hline \multirow[t]{2}{*}{1} & 328 & 0 & 105.5 & 105.5 & 90.0 & 15 & 25 & 160 & 1.82 & 123.2 & 9.2 & 300.9 & 231.7 & NA & -204.6 & NA & -3.4 \\
\hline & 600 & 1 & 105.5 & 105.5 & 90.0 & 15 & 25 & 160 & 1.82 & 123.2 & 8.9 & 292.1 & 197.3 & 110.6 & 148.8 & 2.0 & 2.8 \\
\hline \multirow[t]{3}{*}{2} & 462 & 0 & 90.1 & 90.1 & 90.0 & 22 & 25 & 160 & 1.82 & 111.7 & 9.3 & 271.0 & 228.4 & NA & -37.4 & NA & -0.7 \\
\hline & 93 & 0 & 90.1 & 90.1 & 90.0 & 22 & 25 & 160 & 1.82 & 111.7 & 9.3 & 271.0 & 228.4 & $\mathrm{NA}$ & -406.4 & $\mathrm{NA}$ & -7.3 \\
\hline & 211 & 0 & 90.1 & 90.1 & 90.0 & 22 & 25 & 160 & 1.82 & 111.7 & 9.3 & 271.0 & 228.4 & NA & -288.4 & $\mathrm{NA}$ & -5.2 \\
\hline \multirow[t]{2}{*}{4} & 383 & 0 & 117.6 & 77.9 & 90.0 & 20 & 25 & 160 & 1.82 & 117.6 & 4.2 & 125.6 & 95.0 & NA & 162.4 & NA & 2.9 \\
\hline & 800 & 1 & 77.9 & 77.9 & 90.0 & 20 & 25 & 160 & 1.82 & 102.5 & 8.0 & 207.2 & 199.0 & 592.8 & 564.1 & 20.8 & 20.2 \\
\hline 5 & 800 & 1 & 85.5 & 85.5 & 90.0 & 21 & 25 & 160 & 1.82 & 108.2 & 8.8 & 246.4 & 219.7 & 553.6 & 638.1 & 18.4 & 20.2 \\
\hline 6 & 800 & 1 & 78.4 & 78.4 & 90.0 & 20 & 25 & 160 & 1.82 & 102.9 & 9.4 & 239.7 & 234.7 & 560.3 & 582.0 & 19.6 & 20.1 \\
\hline 8 & 800 & 1 & 81.0 & 81.0 & 90.0 & 20 & 25 & 160 & 1.82 & 104.9 & 7.5 & 205.4 & 188.0 & 594.6 & 678.0 & 20.4 & 22.2 \\
\hline \multirow[t]{4}{*}{9} & 80 & 0 & 79.2 & 79.2 & 90.0 & 20 & 25 & 160 & 1.82 & 103.5 & 8.0 & 211.4 & 182.3 & NA & -313.8 & NA & -6.1 \\
\hline & 42 & 0 & 79.2 & 79.2 & 90.0 & 20 & 25 & 160 & 1.82 & 103.5 & 8.0 & 211.4 & 182.3 & NA & -351.8 & NA & -6.8 \\
\hline & 165 & 0 & 79.2 & 79.2 & 90.0 & 20 & 25 & 160 & 1.82 & 103.5 & 8.0 & 211.4 & 182.3 & NA & -228.8 & NA & -4.4 \\
\hline & 620 & 1 & 79.2 & 79.2 & 90.0 & 20 & 25 & 160 & 1.82 & 103.5 & 8.5 & 222.0 & 211.9 & 398.0 & 417.8 & 13.8 & 14.3 \\
\hline 10 & 476 & 0 & 82.3 & 82.3 & 90.0 & 21 & 25 & 160 & 1.82 & 105.8 & 8.3 & 226.9 & 241.4 & NA & 7.7 & NA & 0.1 \\
\hline$\ldots$ & $\ldots$ & $\ldots$ & $\ldots$ & $\ldots$ & $\ldots$ & $\ldots$ & $\cdots$ & $\ldots$ & & $\ldots$ & & $\ldots$ & $\ldots$ & $\ldots$ & $\ldots$ & $\ldots$ & $\cdots$ \\
\hline
\end{tabular}


TABLE 3 Parameter estimates for critical TTC based on the Probit model

\begin{tabular}{|c|c|c|c|}
\hline \multicolumn{4}{|c|}{ Model: $T^{|c|} C^{\text {crit }} \sim N\left(\overline{T T}_{\text {crit }}, \sigma^{2}\right)$} \\
\hline Parameter & Estimated Value & Standard Error & P-value \\
\hline$\overline{T T}_{\text {crit }}(s)$ & 3.0 & 0.80 & 0.010 \\
\hline$\sigma(s)$ & 0.7 & 0.51 & 0.003 \\
\hline \multicolumn{3}{|c|}{ Log likelihood $=-16.35$} \\
\hline
\end{tabular}


Amir H. Ghods, Frank F. Saccomanno

TABLE 4 - GHR car-following model calibration parameters

\begin{tabular}{|c|c|c|c|c|c|c|}
\hline $\begin{array}{c}\text { Car-following } \\
\text { Parameters }\end{array}$ & $a_{n}^{\max }\left(\mathrm{m} / \mathrm{s}^{2}\right)$ & $d_{n}^{\max }\left(\mathrm{m} / \mathrm{s}^{2}\right)$ & $\hat{d}_{n-1}\left(\mathrm{~m} / \mathrm{s}^{2}\right)$ & $L_{n-1}(\mathrm{~m})$ & $T(\mathrm{~s})$ & $s(\mathrm{~m})$ \\
\hline Normal Following & 1.7 & -3.4 & -3 & 4.9 & 1 & 2 \\
\hline $\begin{array}{c}\text { Following With } \\
\text { Overtaking Desire }\end{array}$ & 1.7 & -3.7 & -2.7 & 4.9 & 1 & 2 \\
\hline
\end{tabular}

$a_{n}^{\max }:$ Maximum desired acceleration of the following vehicle $\left(\mathrm{m} / \mathrm{s}^{2}\right)$

$d_{n}^{\max }:$ Maximum desired deceleration of the following vehicle, $\left(\frac{m}{s^{2}}\right)$

$\hat{d}_{n-1}\left(\frac{m}{s^{2}}\right)$ : Estimation of maximum desired deceleration rate of the lead vehicle $\left(\frac{m}{s^{2}}\right)$

$L_{n-1}(m)$ : Length of the lead vehicle, $(m)$

$T(s)$ : Reaction time of the following vehicle, $(s)$

$s(m)$ : Desired distance to the lead vehicle while standing (front to end) 
TABLE 5 Traffic data information reported from field data as reported in Hegeman (2004)

\begin{tabular}{|c|c|c|}
\hline & Direction 1 & Direction 2 \\
\hline Volume (vph) & 1026 & 471 \\
\hline Truck Percentage (\%) & 7.2 & 5.2 \\
\hline Average speed $(\mathrm{km} / \mathrm{h})$ & 85.9 & 91.8 \\
\hline Standard deviation of speed $(\mathrm{km} / \mathrm{h})$ & 5.8 & 7.2 \\
\hline Minimum speed $(\mathrm{km} / \mathrm{h})$ & 61 & 71.6 \\
\hline Maximum speed $(\mathrm{km} / \mathrm{h})$ & 115.7 & 114.5 \\
\hline
\end{tabular}


TABLE 6 Observed versus simulated overtaking rate and average travel speed for the three simulation model of TRARR, OTSIM, and TWOPAS

\begin{tabular}{|c|c|c|c|c|c|c|c|c|}
\hline \multicolumn{2}{|c|}{ Data source } & \multirow{2}{*}{$\begin{array}{c}\text { Field } \\
\text { Data* }\end{array}$} & \multirow{2}{*}{ TRARR* } & OTSIM & $\begin{array}{c}\text { TWOPAS } \\
\text { (IHSDM) }\end{array}$ & $\begin{array}{c}\text { Absolute Difference } \\
\text { TRARR } \\
\text { vs. Field }\end{array}$ & $\begin{array}{c}\text { OTSIM } \\
\text { vs. } \\
\text { Field }\end{array}$ & $\begin{array}{c}\text { TWOPAS } \\
\text { vs. Field }\end{array}$ \\
\hline $\begin{array}{c}\text { Overtaking rate } \\
(\mathbf{O T} / \mathbf{k m} / \mathbf{h})\end{array}$ & Direction 1 & 52.1 & 109 & 49.8 & 30.5 & 56.9 & 2.3 & 21.6 \\
\cline { 2 - 10 } & Direction 2 & 3.7 & 66 & 7.5 & 2.5 & 62.3 & 3 & 1.2 \\
\hline $\begin{array}{c}\text { Average Travel } \\
\text { Speed (km/h) }\end{array}$ & Direction 1 & 85.9 & NA** & 81.6 & 77.2 & NA & 4.3 & 8.7 \\
\cline { 2 - 10 } & Direction 2 & 91.8 & NA & 87.2 & 84.5 & NA & 4.6 & 7.3 \\
\hline
\end{tabular}

* Reported in Hegeman 2004)

** Not Available (reported to be lower than field data) 
FIGURE 1 Snapshots of overtaking maneuver phases for TTC estimation

FIGURE 2 Site characteristics for video recording (Guido et al. 2013)

FIGURE 3 Perception error distributions estimated from observed accepted gaps

FIGURE 4- Probit regression and distribution of TTC critical gaps for the population of drivers

FIGURE 5- OTSIM simulation schematic diagram

FIGURE 6 Comparing PTSF between OTSIM, TWOPAS, and HCM2010 


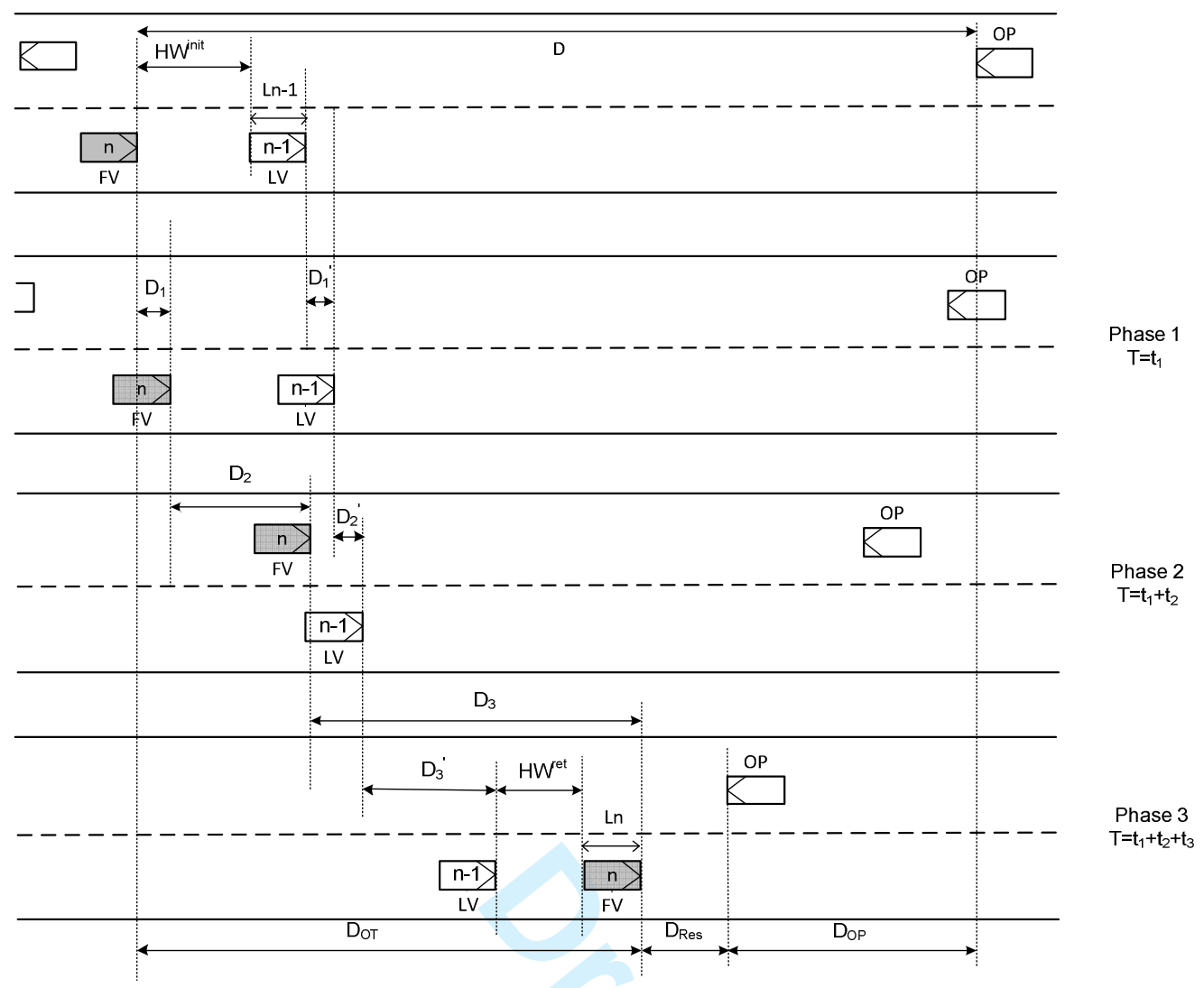




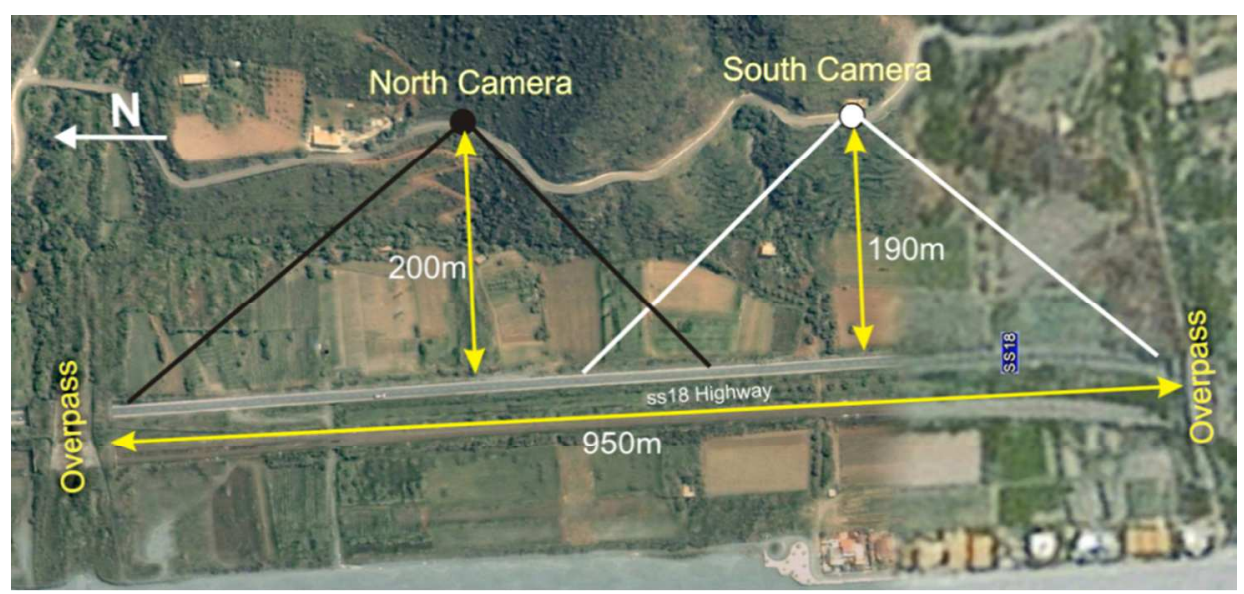



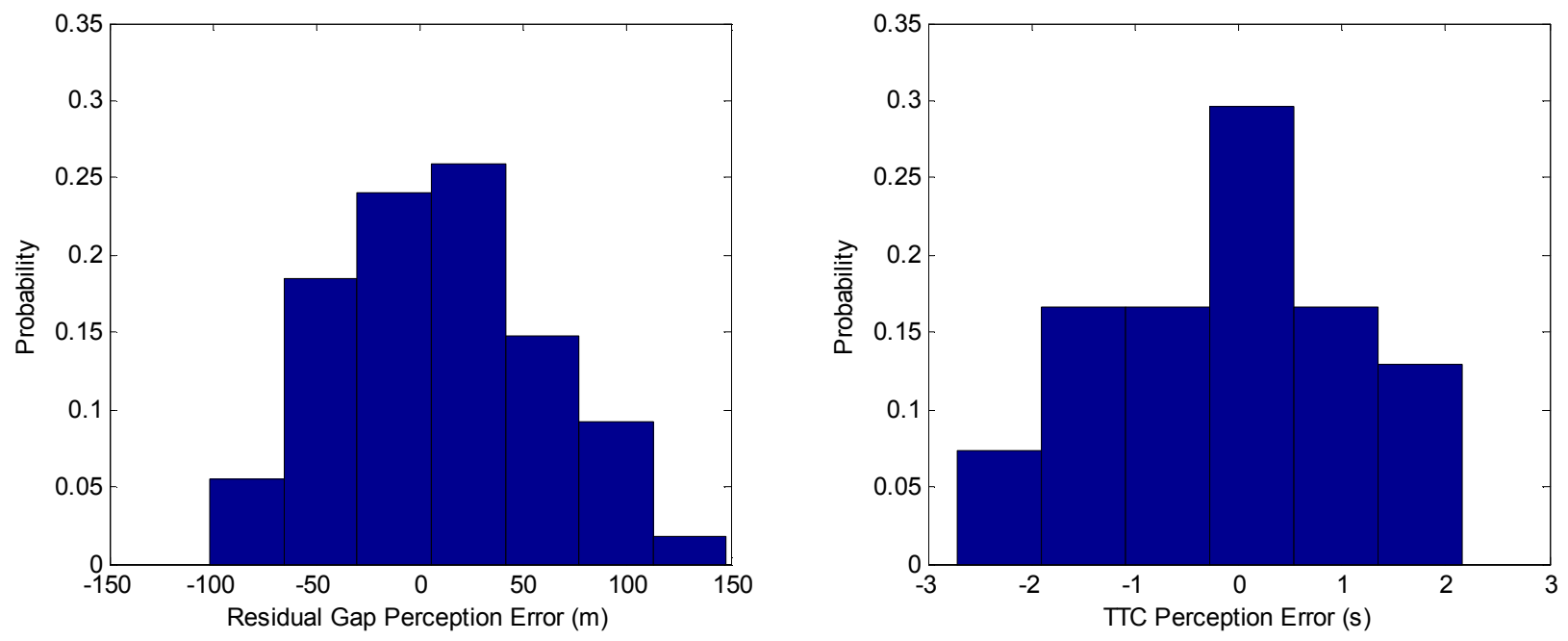


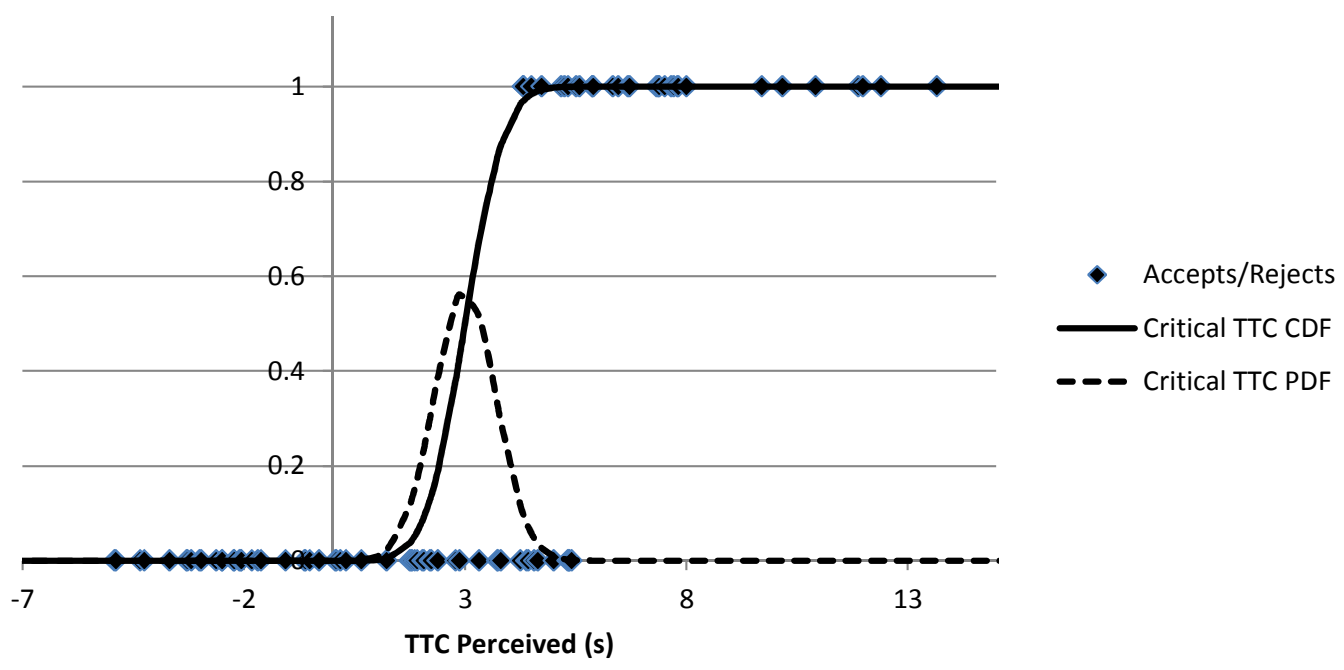




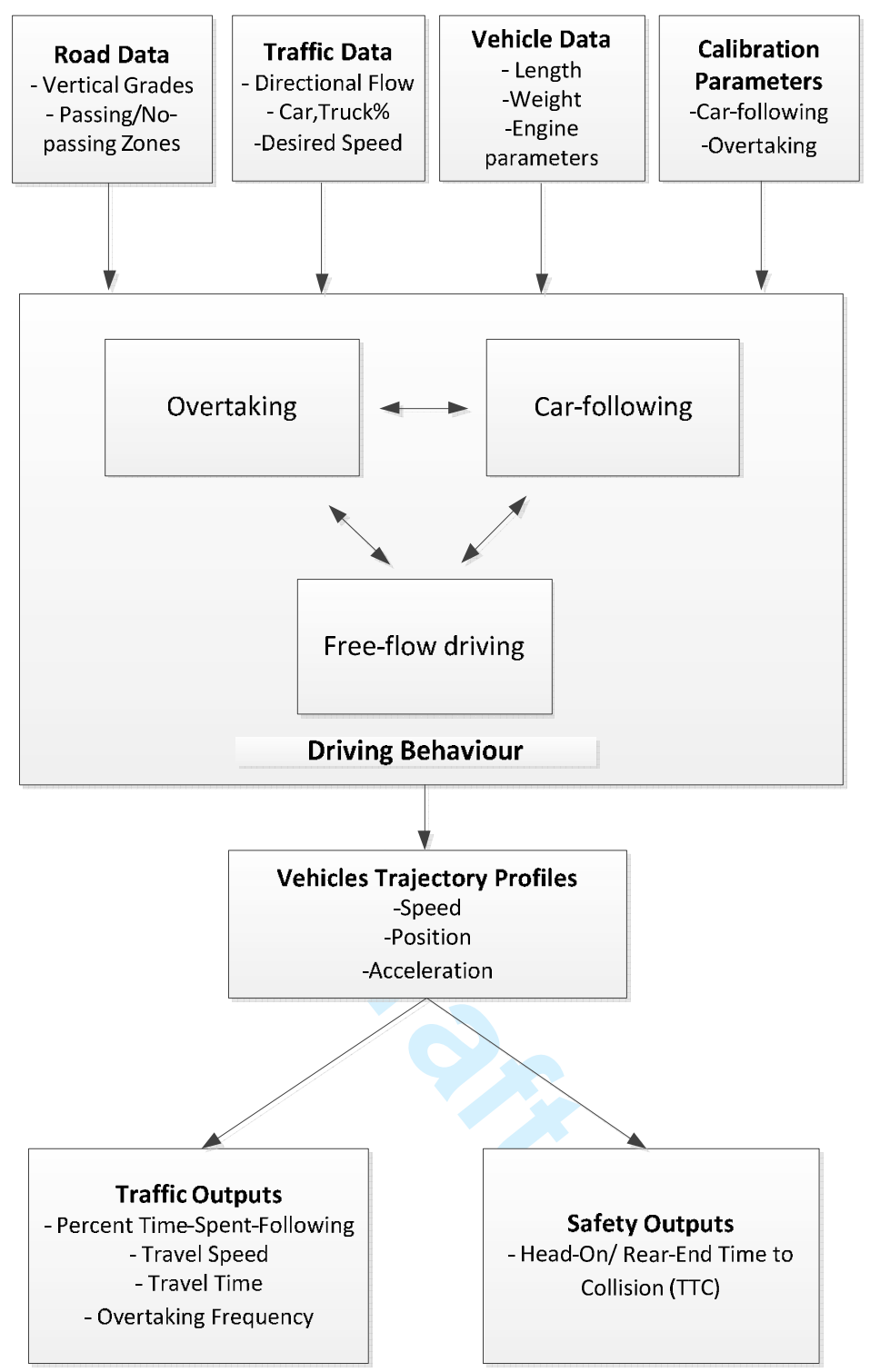



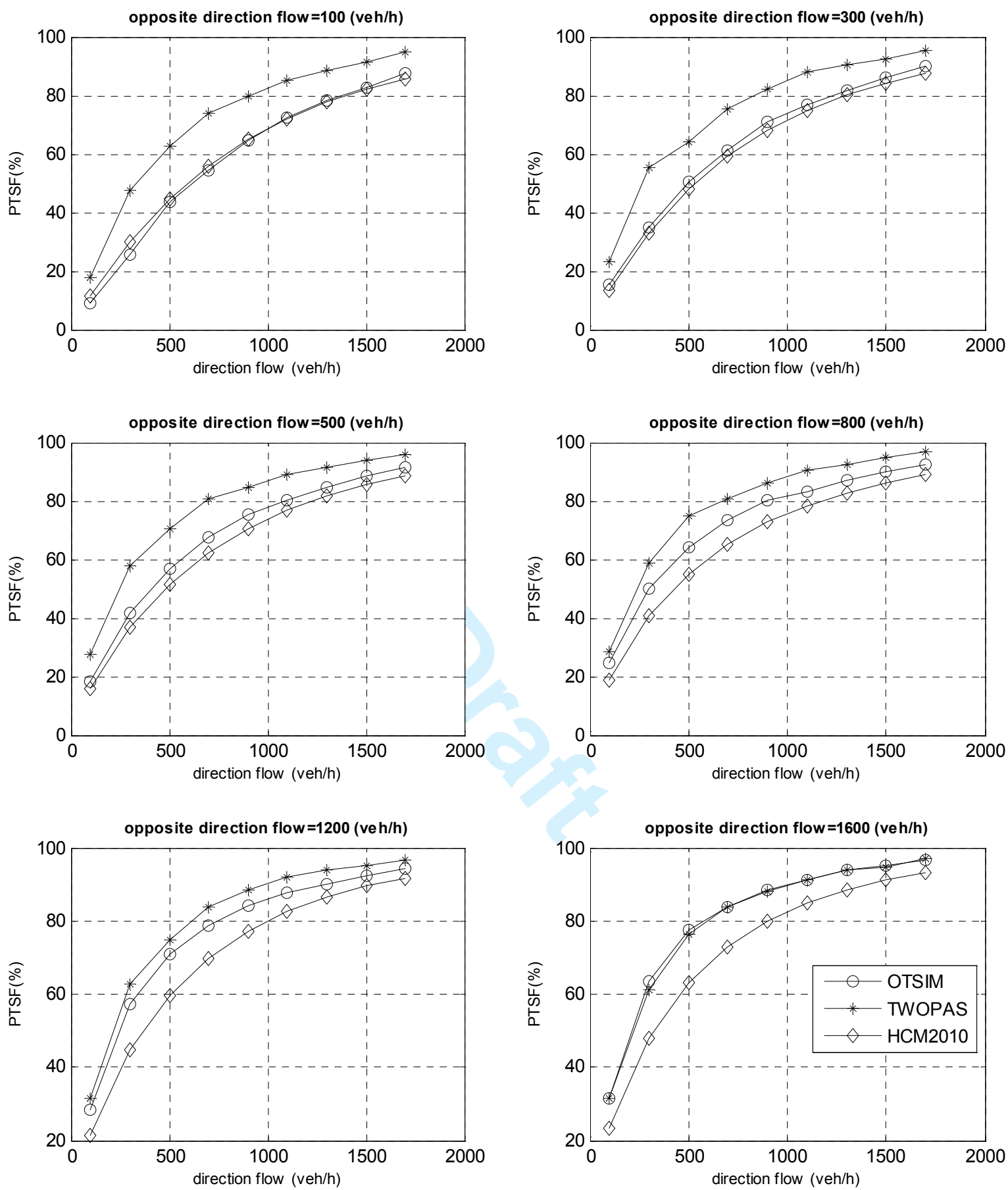